\title{
Advance of modified ELISA and their application
}

\author{
Hejia $\mathrm{Li}^{1, \mathrm{a},{ }^{*}, \dagger}$, Yuhan $\mathrm{Li}^{2, \mathrm{~b},{ }^{*}, \dagger}$ and Yihan $\mathrm{Xia}^{3, \mathrm{c},{ }^{*}, \dagger}$, \\ ${ }^{1}$ Ulink College of Shanghai, China \\ ${ }^{2}$ The Second High School attached to Beijing Normal University \\ ${ }^{3}$ Biochemistry, Department of Chemistry, University of Washington, the United States \\ †These authors contributed equally
}

\begin{abstract}
ELISA (enzyme-linked immunosorbent assay) is a reliable and sensitive method of detection technique. It is commonly used in the daily research of scientific institutions and laboratories world widely. Although ELISA has an acceptable sensitivity and accuracy, when it comes to timely information about urgent detections, conventional ELISA lacks the ability to provide that. In this article, we introduce various types of modifications of ELISA and their applications in many fields, including the clinic diagnosis and the food detection, as well as the introduction of practical ELISA systems.
\end{abstract}

\section{Introduction}

As a common technique used for disease-associated biomarker detection, ELISA is widely adopted in the clinal field for diagnosis of disease and predicts treatment success, and ELISA is also used in non-clinical detection, like in food quality control, environmental and chemical field. In fact of the rapid spread of usage of ELISA in bioanalytical domain, numerous promising modifications of ELISA are introduced in terms of seven parameters of the bio-analytical platform that comes up. In addition to the modification of ELISA, the main goal of modifying ELISA is to apply ELISA for various fields. Clinical diagnosis and food safety inspection are the two main practical uses enumerated in this work. To extend the understanding of the modified ELISA, one specific example provided is the digital ELISA. This work describes and analyses the digital ELISA with its development status and some of its application.

\section{ELISA modifications}

\section{1 sensitivity}

Although ELISA is one of the most sensitive immunoassays available, considerable approaches appear to enhance the performance of ELISA. It is because of the limiting resources, and there is the urgency of improving the sensitivity of ELISA when a low concentration sample exists in order to obtain a wider usage of ELISA. Tons of modification was introduced to elevate its sensitivity, involving in addition of nanoparticles to antibodies in the basic indirect ELISA. For instance, the utilization of DNA to replace the enzyme used in standardized ELISA and the nucleic amplification performed by CRSPR/Cas 13a to achieve the amplification of the signal.

\subsubsection{Nanoparticles-conjugated ELISA}

The standard ELISA consists of the antigen from the sample, antibody binding to the antigen, so the elevation of the signal ability of antibody by attaching antibodies to nanoparticles helps to improve the sensitivity of ELISA. (Fig 1)

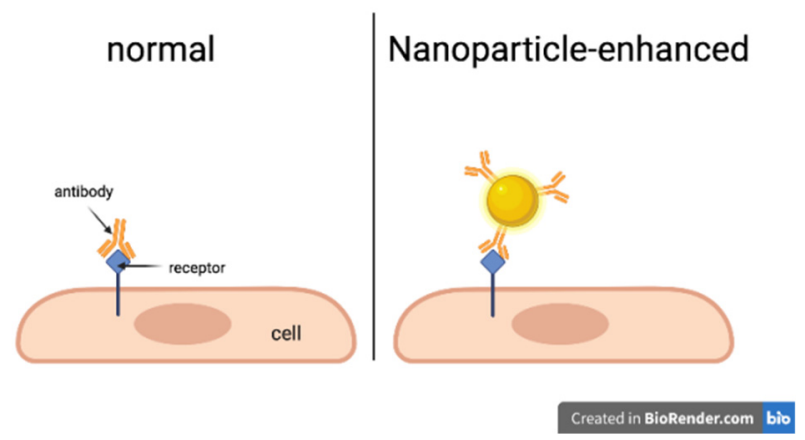

Fig 1. Normal vs. nanoparticle enhanced ELISAs.

In normal ELISA (left), primary antibodies flow freely and bind to their target receptor, which provide limited amount of binding sites for secondary antibodies. In nanoparticle-enhanced ELISA(right), nanoparticles are coated with primary antibodies to increase the number of binding sites for secondary antibodies.

Aiming to increase the sensitivity of ELISA, Margaret M. Billingsley et al. come up with the way of incorporating nanoparticles conjugated antibody into

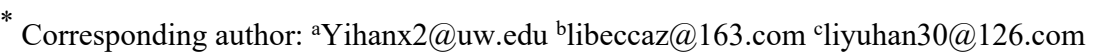


standard ELISA. The primary antibody is decorated in nanoshells for detecting breast cancer cells. Because of the nanoparticles, more secondary antibodies could bind and show a higher resultant signal [1].

Additionally, the use of metal nanoparticles as an antibody carrier could also develop an effective way to improve its sensitivity. Adriano Ambrosi et al., utilizes gold nanoparticles (AuNPs) as carriers for anti-CA15-3HRP (horseradish peroxidase). As a result, it shows an amplification in sensitivity as well as shorter assay time [2]. The novel probe is based on the colloidal gold nanoparticle (AuNP) that is modified with antigens in order to amplify the signal. Also, HRP synthesized used as signal amplification in many cases. Zhou $\mathrm{Yu}$ et al., have modified the probe with goat anti-mouse $\operatorname{IgG}$ and synthesize HRP. Based on the prob developed, the enhanced ELISA is developed. The newly synthesized probe bound with monoclonal antibody $(\mathrm{McAb})$ which bound by coated BSA-ITCBE-Pb(II) on the plate and $\mathrm{Pb}$ (II) in the sample [3]. The HRP acts as a signal amplification to generate the optical signal to be measured. Due to the sphere shape of AuNP, more HRP conjugated comparing to $\mathrm{IgG}$, result in a higher sensitivity. Both ways of similar application of AuNP indicates that metal nanoparticles, due to its large interaction area, enhance the basic ELISA that have flat and less-effective conjugation of signals, lead to an elevated sensitivity

\subsubsection{Nucleic amplification ELISA}

Another route to improve the sensitivity of ELISA is the use of DNA or RNA amplification method. Based on the knowledge that nucleic acid attached with optical signal it also plays a role in signaling response for detection. Qian Chen's group utilized the double-standard DNA containing T7 promoter sequence to replace the enzyme (horseradish peroxidase), T7 polymerase recognizes the promoter and proceeds transcription to produce singlestandard RNA (ssRNA). After that, they designed CRISPR/Cas 13a system, which trans-cleaves ss-RNA. Short ssRNA reporter labeled with fluorophore and quencher group at both ends can be cleaved by transcleavage activity, leads to the amplification of signal [4].(Fig 2) This process is named CRISPR/ Cas 13a signal amplification linked immunosorbent assay (CLISA), which is confirmed at least $10^{2}$-fold more sensitive than conventional ELISA in detecting inflammatory factors, tumor markers etc [4].
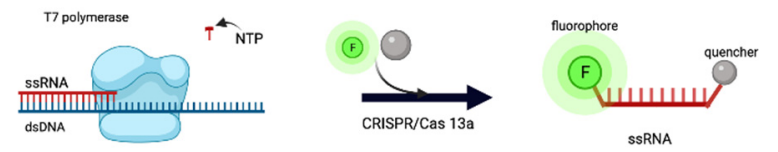

Fig 2. CRISPR/Cas $13 \mathrm{a}$
T7 polymerase synthesizes ssRNA from dsDNA with NTP addition for modification in ssRNA. Then, CRISPR/Cas 13a cleaves ssRNA into short RNA fragment and add fluorophore and quencher group on both end as reporter signal.

Nucleic acid sequence-cased amplification (NASBA) is commonly used to continuously amplify nucleic acid bases on primer-dependent technology. Therefore, with the ability to amplify, nucleic acid sequence-based amplification with enzyme-linked immunosorbent assay (NASBA-ELISA) was developed for more sensitive ELISA. In order to amplify nucleic acid, a specific primer would be designed targeting RNA genome for amplification, producing amplicons. The amplicons were facilitated for probes then detected by a microplate reader. For instance, Gill P et al., performed this method for the detection of Mycobacterium tuberculosis. By introducing primer targeting on bacterial RNA, the amplification is made by isothermal digoxigenin (DIG)-labeling NASBA process. The accumulation of DIG-labeled RNA amplicons was hydrolyzed with a specific DNA probe that immobilized on the plate. The DNA-RNA hybrids were then detected [5]. The amplification by NASBA increases the sensitivity and specificity. Furthermore, for genotype II Grass carp reovirus (GCRV-II) detection in China, Zeng W et al., also comes up with NASBA-ELISA method for overcoming the problem of low sensitivity [6].

\section{2 specificity}

As one of the significant parameters for the clinical analytical platform, specificity contributes to validating the quality of ELISA outcomes. Different from sensitivity, which mainly concentrates on correctly identify disease, specificity measure the ability to correctly identify people without the disease, inhibiting non-specific binding. In order to improve the specificity of ELISA, numerous modifications including Ethanol vortex ELISA (EVELISA) and ZEDIII-based ELISA.

\subsubsection{EVELISA}

EVELISA is commonly used in the diagnosis of Johne's disease (JD) in dairy cattle. This test is objected to the detection of anti-MAP antibodies in milk. Due to the various environmental mycobacteria, more than $70 \%$ of the sample were identified as false positive [7]. Antibodies in the sample reacted in high activity with antigens of various environment mycobacteria, showing cross-reaction antibodies in the sample. The crossreactive antibodies of MAP in the sample were highly inhibited by the use of Mycobacterium phlei antigen for antibody absorption [7]. Ashutosh Washwa et al., also choose EVELISA to test and optimize the concentration of antigens, milk and secondary antibody for segregating MAP-infection herds [8]. By using ethanol extract of MAP, EVELISA performs a basis for a higher sensitivity diagnostic test with a higher level of specificity than the current commercial ELISA test. 


\subsubsection{ZEDIII-based ELISA}

In order to relieve the epidemic of Zika virus (ZIKV) and Dengue Virus (DENV), which are often co-epidemic, numerous amounts of exercise have been introduced by scientists. Their high protein-sequence homology of flaviviruses, ZIKV and DENV had highly cross-relative against each other's antigen(s). [9] Denis et al., observed a ZEDIII-based ELISA using Domain III of the ZIKV Envelope (ZEDIII) sequence. ZEDIII sequence is virusspecific, thus it is purified for specific detection of ZEDIII-induced IgG [9]. This kind of recombinant domain of ZEDIII-based ELISA distinguishes between past and current DENV and ZIKV infections. The challenge of serological detection of ZIKV, which has highly cross-reactivity against the related virus in detection. The ZEDIII-based ELISA assay showed 90\% specificity and $92 \%$ sensitivity according to Jessica and her fellows.

\subsection{Other parameters}

\subsubsection{Rapid}

Aside from being sensitive and specific, the lengthy incubation of ELISA is also a key parameter for ELISA to improve for detecting disease. The faster result contributes a longer time for doctors to outline a treatment. Such techniques particularly serve as the early detection of fatal illness. Samira et al., manufactured a combination of microspheres and microfluidic disks to perform sandwich ELISA [10]. This mixing mechanism increases the molecular contact between target analyte molecule and microsphere, thus leads to more effective detection. Along with lower detection limits, the incubation time lengthy deduce from 2 hours to 5 minus. This huge success in enhancing the rapid of ELISA by modifying two mechanisms together contribute an earlier detection result, which gives a promising future in the early detection of fatal illness.

\subsubsection{User-friendliness}

Scientists and medical companies always longed for convenient equipment that releases the tasks of the scientist. Automation of equipment is one of the demands for ELISA in further development of modifying ELISA technique. Conventional ELISA is a series of mixing, washing, and incubation steps. The technology of microfluidic devices, the chance to release scientists' hands and promise the accuracy can be taken into consideration. The immobilization of biomarkers takes place in the channel, and due to the miniaturized size, the assay could perform in automation by the electrical or magnetic forces. AlFaqheri et al., introduced a microfluidic compact disk (CD) platform that presents an easy to fabricate air and liquid check valves rather than a wide range of active and passive valving methods that is tested in other microfluidic platforms that is too complex to analyze [11].

\subsubsection{Affordability}

After the sensitivity and specificity improvement, in order to benefit the population with ELISA detection machine, the modification to provide a more affordable price is also one of the focuses. Enzyme-linked immunosorbent spot (ELISpot) contains the advantage of affordability. ELISpot will yield information about a number of cells secreting cytokine of interest. IFN-g-based assay is one of the most popular forms of ELISpot technique. IFN-g is secreted by activated T cells abundantly. The IFN-g assay could be widely used in vaccine discovery by the immune response quantity. High sensitivity, multiple target analytes, and affordable prices are some of the main advantages of this technique [12].

\section{MODIFIED ELISA APPLICATION}

Since the ELISA can be used to detect the presence of particular antigens or other substances, it is widely used in plenty of areas including clinical diagnosis and food safety inspection.

\subsection{Clinical diagnosis}

To diagnose the disease accurately, doctors need to analyze the elements collected from patients' bodies besides just asking their symptoms. In most cases, a specific antigen is a key to indicate the occurrence of one disease because of the specificity. However other substances such as proteins can also be used for detection. In this section, several clinical diagnosises using ELISA will be present.

\subsubsection{Chikungunya(CHIK) diagnosis}

Chikungunya (CHIK) fever is an acute arthropod-borne viral illness in which the pathogen is CHIK virus. The explosive epidemic of CHIK emerged major public health problem in India and Indian Ocean islands. It is a good example of ELISA application in clinical diagnosis. The original diagnosis was essentially based on virus isolation, IgM ELISA and reverse transcriptase polymerase chain reaction (RT-PCR). However, its high cost and long time for waiting the result led to an alternative way for early clinical diagnosis. The advanced sandwich ELISA employed rabbit and mouse anti-CHIK IgG antibodies as the capture and the detector antibodies, respectively.

The particular strain for the research was an India strain CHIKV ISW HYD06. To produce antibodies against CHIKV, purified CHIKV antigen mixed with equal volume of Freund's complete adjuvant (FCA) to immunize rabbit New Zealand strain). At the same time, a group of 3 to 4-week-old Swiss albino mice was also immunized subcutaneously with $100 \mu \mathrm{g}$ of purified CHIK viral antigen and an equal volume of FCA. Thus, the production was subjected to titration by indirect microtiter plate ELISA using the CHIK viral antigen.

The purified CHIK viral protein was diluted to 300 $\mathrm{ng} / \mu \mathrm{L}$ in $0.1 \mathrm{~mol} / \mathrm{L}$ carbonate buffer, $\mathrm{pH} 9.6$, and used for 
coating in 96-well microtiter plate $(100 \mu \mathrm{L} /$ well $)$ and incubated at $4^{\circ} \mathrm{Cfor}$ overnight. The coated wells were washed once with PBS $(1 \times$ PBS $)$ and then blocked with $3 \%$ Bovine serum albumin (BSA) in $1 \times$ PBS followed by incubation at $37^{\circ} \mathrm{C}$ for $2 \mathrm{~h}$. After washing with $1 \times \mathrm{PBS}$, $100 \mu \mathrm{L}$ of rabbit and mouse HIS was titrated at an optimum dilution ranging from 1:1000 to $1: 1024000$, which were added in the wells and incubated at $37^{\circ} \mathrm{C}$ for $1 \mathrm{~h}$. The plate was incubated with anti-rabbit and antimouse HRP conjugate at a dilution of 1:2000 made in 3\% BSA followed by washing steps. The reaction was developed with $100 \mu \mathrm{L}$ of tetramethylbenzi dine (TMB) substrate and kept at room temperature (RT) for $5 \mathrm{~min}$. The peroxidase reaction was terminated with $100 \mathrm{~L}$ of 1 $\mathrm{N} \mathrm{H} 2 \mathrm{SO} 4$, and the absorbance was read at $490 \mathrm{~nm}$ in an ELISA reader.

To determine the optimum working concentration of both the rabbit and mice HIS, titration was performed through indirect double antibody sandwich microplate ELISA. The optimum titer of rabbit and mice HIS to be used as capture and detector antibodies in the antigen capture ELISA was found to be 1:32 000 and 1:128 000, respectively.

Combined with ELISA and real time PCR, the result showed an accordance of $96 \%$. Moreover, it had high sensitivity (95\%) and specificity (97\%). This study was able to detect the presence of viral antigen as early as the second day of fever and, thus, it can be very useful for the early clinical diagnosis of CHIK with acute-phase patient serum and CSF samples [13].

\subsubsection{Allergy diagnosis}

Toshinori Ohashi and his team developed a practical and more sensitive micro-ELISA system for allergy diagnosis. Based on the previous method for allergy diagnosis, they modified the links of BSA-biotin-avidin so they were immobilized on the polystyrene surface by physisorption, ameliorated non-specific adsorption. Another improvement was the stopped-flow conditions of the enzymatic reaction to produce dye molecules.(Fig.3)

In their micro-ELISA system including fluid and optical components, the samples and reagent are stocked in tubes on an automated rotating stage. In the next automated stage, a specific volume of the sample and reagents would be sucked. The samples and reagents were then driven to a bead-packed area at a determined flow rate by the syringe pump. The length of the tube set shorter to $20 \mathrm{~mm}$ to prevent broadening of the dilute sample to decrease the sensitivity. Micro beads ( $45 \mathrm{~mm}$ ) were introduced to the micro channel and a precise $5 \mathrm{~mm}$ long bead-packed area was formed by introducing more beads and flushing the extra length with a syringe and valve. (Fig.3)

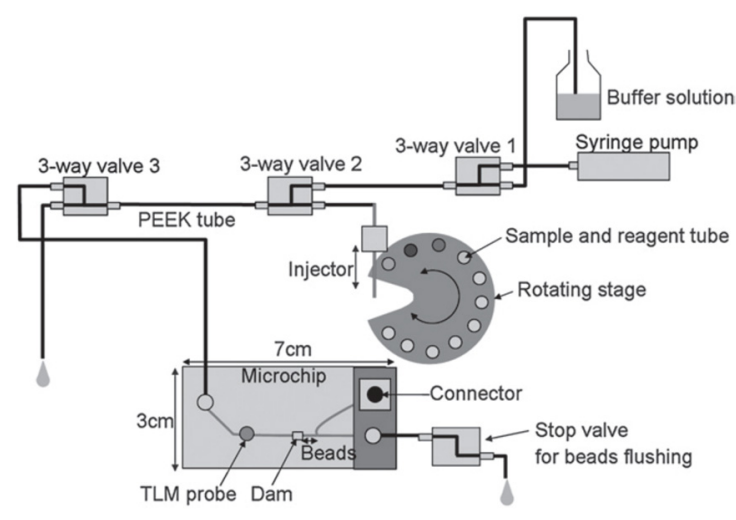

Fig.3 block diagram of micro-ELISA system.[14]

The measurement protocol for allergy was similar to the traditional way, including analyte aspiration, antibody aspiration, substrates aspiration and enzymatic reaction. The process from the introduction of the bead to bead removal only lasts 12 minutes. BSA buffer solution $(0.2 \%)$ was used as the micro-ELISA system carrier solution. In their research, Cedar pollen allergen Cry j1, Cedar pollen allergen Cry j2, biotin-labeled cedar pollen allergen Cry $\mathrm{j} 1$ and biotin-labeled cedar pollen allergen Cry j2 were used for allergens.

This system was tested using human serum samples from 85 allergy patients. Signal intensity was measured by the micro-ELISA system, and the concentration was calculated by utilizing 4-parameter logistics for improving the accuracy. Total IgE and Cedar specific IgE were selected. For comparison, the same serum samples were measured by the CAP method (conventional method).

The advantage for this micro-ELISA system was more sensitive and faster. It was demonstrated using a smaller sample volume (1/10) and a shorter analysis time (1/20). All the processes were automatically applied with a micro-fluid system, extending the applicability to real clinical diagnostic situations [14].

\subsection{Food safety inspection}

In the last few decades, the rapid development of modified ELISA has become a widely used technique in food detection because of its specificity and sensitivity. Both monoclonal and polyclonal antibodies can be used in ELISA for food analysis. Polyclonal antibody is able to recognize a mixture of different epitopes of the antigens, more tolerance to small changes in the nature of antigen while monoclonal antibody has defined biological activity, consistent specificity and their production are not limited [16]. The most used immunoassays kits for food component identification are ELISA and lateral flow tests. The use of ELISA technique on food identification and detection would be illustrated by examples of application in the milk and meat industry [15].

\subsubsection{Application in milk products}

In the milk industry, health issues need to be considered from the production of the raw milk from cows to the storage of the final products. Bovine respiratory syncytial 
virus (BRSV) is one of the most important causes of respiratory disease in beef and dairy cattle. Fever, coughing, respiratory distress, and a marked drop in milk production are the main symptoms. M. Elvander, S. Edwards, IS. Näslund, N. Linde evaluated the NVI ELISA detection for BRSV in serum and milk. The results showed that there was a good correlation between the ability to detect antibodies in serum and the ability to detect them in milk. There was a clear distinction between antibody levels in diseased and healthy herds. The NVI ELISA is a rapid and reliable test for detecting antibodies to BRSV in milk, bulk milk, and serum samples [17].

Aflatoxins (AFs) are extremely toxic substances produced by certain species of Aspergillus, especially Aspergillus flavus, A. parasiticus, and A. nomius that contaminate plants and its products. AFM1 forms in the liver and it is secreted into milk in the mammary gland of dairy cows. AFM1 intake, even at low concentrations, causes a significant risk to human health, especially to children who are the major consumers of milk. A China research group evaluated the occurrence of milk and milk products contamination with AFM1 in Northeast of China by ELISA using monoclonal antibodies. Of 135 milk samples tested, 55 (41\%) samples contained AFM1 at levels that ranged from $0.32-0.50 \mathrm{ng} / \mathrm{ml}, 24(18 \%)$ samples contained $0.16-0.32 \mathrm{ng} / \mathrm{ml}$, and $18(13 \%)$ samples contained $0-0.16 \mathrm{ng} / \mathrm{ml}$; in 38 (28\%) samples AFM1 was not detected. Therefore, precautions were necessary for minimizing the AFM1 contamination in milk and milk products in Chin [18].

The aminoglycoside antibiotics are used for the treatment of Gram-negative and Gram-positive infections. However, the presence of neomycin in animalorigin food is potentially hazardous to human health. For consumer protection, it is important to monitor neomycin residual levels in biological matrixes. The advanced ELISA was developed for the fast screening of neomycin in milk samples with high sensitivity, specificity and minimised matrix interference. The optimised ic ELISA could be finished in 55 minutes [19].

\subsubsection{Application in meat}

Two main purposes using ELISA in the meat industry is to identify the animal species so that to prevent the adulteration and detect the pathogen in meat.

For example, Martin, Chan, and Chiu (1998) carried out successfully a quantitative evaluation of pork adulteration in raw ground beef by ELISA [20]. It can be also applied for non-meat proteins, such as soybean proteins which is the potential health threat for Allergy sufferers. Macedo-Silva, Shimokomaki, Vaz, Yamamoto, and Tenuta-Filho discovered soy proteins in hamburgers containing beef, chicken and swine meat. Consequently, they obtained polyclonal antibodies against these proteins and quantified soy protein in these meat products by ELISA [21].

Other contaminants such as viruses, bacteria and antibacterial residues contributed to the concerns about food safety. A broad spectrum antimicrobial agent Chloramphenicol (CAP) was used against many Gram negative and Gram positive bacteria. Some undesirable effects in humans had been indicated so many countries have banned its use in food animals. H. Tajik, S.M. Razavi-Rouhani, M.R. Pajouhi, R. Mahmoudi, A. Haghnazari investigated CAP residue in 160 broiler chickens carcasses collected from three provinces of Iran by Four Plate Test (FTP), ELISA and HPLC. The ELISA assay showed that out of 28 positive samples in FPT, 22 liver, 21 kidney and 14 muscle samples were positive for CAP. ELISA analyses demonstrated that the minimum and maximum levels of 0.54 and $155.2 \mathrm{ng} / \mathrm{g}$ were detected in the kidney and liver, respectively. The data showed that despite the prohibition of CAP application in food animals including poultry, the CAP residue was detectable [22].

The results for detection can also contribute to some regulatory agencies and help pesticide residue analysis. Some companies (Neogen Corporation, Zeu Inmunotec, ELISA Systems and Tepnel, among others) commercialize immunochemical diagnostic kits for the rapid detection and quantification in food [15].

\section{Practical ELISA system}

\subsection{Digital ELISA}

\subsubsection{Introduction}

The sensitivity of protein assays still lags behind nucleic acid assays, such as polymerase chain reaction (PCR). Isolation and detection of single protein molecules offer a promising method for measuring very low concentrations of proteins

\subsubsection{Research status}

Single Molecule Array (SIMOA) is aside from known as digital ELISA. It uses a femtoliter-sized array of reaction chambers to segregate and ascertain distinct individual enzyme molecules. The point is to use the ability to capture and detect individual enzymes to detect different protein molecules labelled by enzymes. In the first step of this single-molecule immunoassay, the sandwich antibody complex is formed on a $2.7 \mu \mathrm{m}$ diameter microsphere. The binding complex is labelled with an enzyme, just like a traditional bead-based ELISA. When testing samples containing very low concentrations of protein, the ratio of protein molecules to beads is small and usually less than $1: 1$. For example, if 3000 molecules in $0.1 \mathrm{~mL}$ of protein were captured and tagged on 200,000 beads, then $1.5 \%$ of the beads would contain protein molecules and $98.5 \%$ of the beads would have no protein molecules. These low numbers of enzyme markers cannot be detected using standard detection techniques because each enzyme produces a fluorophore that diffuses over a large number of measurements, typically $0.1-1 \mathrm{~mL}$, requiring thousands of enzyme markers to produce a fluorescence signal higher than the background.

SIMOA needs to be able to detect enzyme markers at very low concentrations by limiting the fluorophore 
produced by a single enzyme to a very small volume, resulting in high local concentrations of fluorescent product molecules. To achieve this position in the immunoassay, in the second step, the beads are placed into a series of spike-sized holes. Approximately 50,000 Wells were drilled for the project, measuring $2 \mathrm{~mm}$ wide, $4.5 \mu \mathrm{m}$ in diameter, and $3.25 \mu \mathrm{m}$ in depth. The loaded array is then sealed on a rubber gasket in the presence of luciferase substrate droplets, isolating each bead in the ascending chamber. In a $50 \mathrm{fl}$ reaction chamber, beads containing a single enzyme labelled immune complex produced a localized high concentration of the fluorescent product. By using standard microscope optics to acquire time-lapse fluorescence images of the array, beads associated with individual enzyme molecules can be distinguished from those associated with non-enzyme molecules. Protein concentration in the test sample is determined by calculating the number of pores containing beads and fluorescent products relative to the total number of pores containing beads. With SIMOA, the concentration is determined digitally rather than using the total analog signal; therefore, the use of SIMOA to detect a single immune complex is called a digital ELISA.

\subsubsection{Comparison between Digital ELISA and early Elisa methods}

The sensitivity of SIMOA assay was $0.62 \mathrm{pg} / \mathrm{mL}$ and ELISA assay was $78.0 \mathrm{pg} / \mathrm{mL}$. So, we found that SIMOA was more sensitive than ordinary ELISA tests. The highly sensitive SIMOA technique merits further study in a larger cohort of patients.

The advantage of ELISA is that it only requires a simple procedure, and under the simple procedure, it is also provided with high specificity, high sensitivity and high efficiency. Because ELISA is just simply based on antigen-antibody reaction. Also, about the safety, because ELISA does not require the radioactive substances and numerous organic solvent, it is safe and eco-friendly. Last, the reagents are in a low cost, so one of the strengths of ELISA, compared with other assays, is cheap. [26]

The digital ELISA contains the same advantages and even better. Even though the whole technology seems more complicated, digital ELISA only needs to use a relatively simple set of equations based on biomolecular interactions for each step of the assay. Moreover, the limit of detection has been improved. The limit of detection of PSA is very low. Many of the current detection methods are not effective, but the digital ELISA exhibits a 3-log improvement in sensitivity compared to the most sensitive commercially available PSA assays. In addition, the accuracy of Digital ELISA can be ensured while the sensitivity is improved. Digital ELISA is a method that literally counts the number of molecules during detection, so provides an excellent way to compare the number of molecules predicted by theory and those detected in experiments. The theoretical results of Digital ELISA are basically the same as the actual results. And the automation of digital ELISA is also an advantage. [24]

\subsubsection{Applications}

The recent increase in global incidence and severity of Clostridium difficile infection (CDI) is a major concern. CDI currently consumes significant resources in diagnosis, treatment and infection control, and accurate diagnosis remains the cornerstone of effective management.

Toxin detection plays an important role in CDI diagnosis, but the current methods of toxin detection are not perfect. Traditional qualitative toxicity immunoassay is widely used, but its LODS is high and its sensitivity is poor. In view of these limitations, the field is poised to develop a simple toxin detection test that combines high analytical sensitivity with clinical specificity for toxin detection.

The ability to isolate and detect individual protein molecules greatly increases the sensitivity to detect the concentration of FG protein per milliliter, typically up to 1000 times more sensitive than traditional ELISA. Digital ELISA has demonstrated high accuracy and reproducibility in detecting low levels of proteins in highly complex matrices such as cell culture supernatants, serum, plasma and cerebrospinal fluid (CSF). The SIMOA method is suitable for the determination of protein in feces. The higher clinical specificity of SIMOA offers the potential to improve diagnostic and management approaches.

Also, Digital ELISA (SIMOA), within higher sensitivity, it is able to detect prostate cancer, Crohn's disease, and neurological disorders. It is able to measure neurological conditions at their very earliest stages. [25]

\subsubsection{Frontiers}

The antibody is difficult to produce and needs a huge amount of labor which causes it to be expensive. Also, the antibody is fragile that it has to be saved in a special temperature to be more specific, cold storage, and has a storage life because the antibody is a protein. Last but not least, insufficient blocking of immobilized antigen results in false consequences. There is a high possibility of false positive or negative. Also, for digital ELISA, because is a single molecule detection, it needs to eliminate the molecular interactions that come with numerous cells, so a lot of noise has to be eliminated.

\subsection{Plasmonic ELISA}

\subsubsection{Research status}

In recent years, plasmonic immunoassay has been developed by combining traditional enzyme-linked immunosorbent assay (ELISA) with nanomaterials. Compared to other immunoassays, plasmonic immunoassays are highly sensitive and allow open-eye reading without the need for the use of complex instruments. Metal nanoparticles, such as gold and silver nanomaterials, are widely used in plasmonic Nanosensors due to their excellent local surface plasmonic resonance (LSPR) properties. In plasmonic 
immunoassay, an enzyme-labeled antibody catalyzes the formation of a product from its substrate, which triggers the aggregation or shape change of the nanomaterial. [23]

\subsubsection{Applications}

Plasmonic immunoassay has been widely used in clinical diagnosis, environmental pollution monitoring and food safety detection. [23]

\subsubsection{Frontier}

Robust, ultra-fast and highly stable AuNPs aggregation in colorimetric analysis remain a challenge due to the dynamic aggregation process of AuNPs. In addition, in order to quantitatively test molecular targets, relatively complex and large instruments are needed to measure the spectra of nanomaterials as well as the plasmonic immunoassay. The inconveniences associated with these instruments make them unsuitable for point-of-care analysis. Therefore, there is an urgent need to develop a portable, inexpensive and easy-to-use plasmonic immunoassay reader for immediate detection (POCT) diagnosis.

\section{Conclusion}

Modified ELISA is undoubtedly the best choice for detection small particles like antigens in clinical detection, bacteria in some food safety tests. In our research, the improvement of ELISA with the development of ELISA application in different regions indicate the advanced modified ELISA techniques have the characteristics of sensitive, accurate and practical.

ELISA is already a proven biosensor, but it is less accurate and cost effective than other methods such as PCR for a particular test. Therefore, the use of ELISA in conjunction with other technologies can be more effective, as in the case of Digital ELISA and Plasmonic ELISA, which have higher precision.

\section{References}

1. M.M. Billingsley, R.S. Riley, E.S. Day, Antibodynanoparticle conjugates to enhance the sensitivity of ELISA-based detection methods, PLOS ONE 12(5) (2017) e0177592.

2. A. Ambrosi, F. Airò, A. Merkoçi, Enhanced Gold Nanoparticle Based ELISA for a Breast Cancer Biomarker, Analytical Chemistry 82(3) (2010) 11511156.

3. Y. Zhou, An enhanced ELISA based on modified colloidal gold nanoparticles for the detection of $\mathrm{Pb}(\mathrm{II})$, Biosensors \& bioelectronics v. 26(no. 8) (2011) pp. 3700-3704-2011 v.26 no.8.

4. Q. Chen, T. Tian, E. Xiong, P. Wang, X. Zhou, CRISPR/Cas13a Signal Amplification Linked Immunosorbent Assay for Femtomolar Protein Detection, Analytical Chemistry 92(1) (2020) 573577.
5. P. Gill, R. Ramezani, M.V.P. Amiri, A. Ghaemi, T. Hashempour, N. Eshraghi, M. Ghalami, H.A. Tehrani, Enzyme-linked immunosorbent assay of nucleic acid sequence-based amplification for molecular detection of M. tuberculosis, Biochem Biophys Res Commun 347(4) (2006) 1151-1157.

6. W. Zeng, W. Yao, Y. Wang, Y. Li, S.M. Bermann, Y. Ren, C. Shi, X. Song, Q. Huang, S. Zheng, Q. Wang, Molecular detection of genotype II grass carp reovirus based on nucleic acid sequence-based amplification combined with enzyme-linked immunosorbent assay (NASBA-ELISA), J Virol Methods 243 (2017) 92-97.

7. M.C. Scott, J.P. Bannantine, Y. Kaneko, A.J. Branscum, R.H. Whitlock, Y. Mori, C.A. Speer, S. Eda, Absorbed EVELISA: a diagnostic test with improved specificity for Johne's disease in cattle, Foodborne Pathog Dis 7(11) (2010) 1291-1296.

8. J.P.B. Ashutosh Wadhwa, Todd M. Byrem, Traci L. Stein, Arnold M. Saxton, C.A. Speer, and Shigetoshi Eda, Optimization of Serum EVELISA for Milk Testing of Johne's Disease, Foodborne Pathog Dis 9(8) (2012) 749-754.

9. J. Denis, S. Attoumani, P. Gravier, B. Tenebray, A. Garnier, S. Briolant, F. de Laval, V. Chastres, G. Grard, I. Leparc-Goffart, B. Coutard, C. Badaut, High specificity and sensitivity of Zika EDIII-based ELISA diagnosis highlighted by a large human reference panel, PLOS Neglected Tropical Diseases 13(9) (2019) e0007747.

10. S. Hosseini, M.M. Aeinehvand, S.M. Uddin, A. Benzina, H.A. Rothan, R. Yusof, L.H. Koole, M.J. Madou, I. Djordjevic, F. Ibrahim, Microsphere integrated microfluidic disk: synergy of two techniques for rapid and ultrasensitive dengue detection, Sci Rep 5 (2015) 16485.

11. W. Al-Faqheri, F. Ibrahim, T.H.G. Thio, M.M. Aeinehvand, H. Arof, M. Madou, Development of novel passive check valves for the microfluidic $C D$ platform, Sensors and Actuators A: Physical 222 (2015) 245-254.

12. A.C. Karlsson, J.N. Martin, S.R. Younger, B.M. Bredt, L. Epling, R. Ronquillo, A. Varma, S.G. Deeks, J.M. McCune, D.F. Nixon, Comparison of the ELISPOT and cytokine flow cytometry assays for the enumeration of antigen-specific T cells, J Immunol Methods 283(1-2) (2003) 141-153.

13. Asensio L, González I, García T, et al. Determination of food authenticity by enzyme-linked immunosorbent assay (ELISA)[J]. Food control, 2008, 19(1): 1-8.

14. Ohashi T, Mawatari K, Sato K, et al. A micro-ELISA system for the rapid and sensitive measurement of total and specific immunoglobulin $\mathrm{E}$ and clinical application to allergy diagnosis[J]. Lab on a Chip, 2009, 9(7): 991-995.

15. Asensio L, González I, García T, et al. Determination of food authenticity by enzyme-linked 
immunosorbent assay (ELISA)[J]. Food control, 2008, 19(1): 1-8.

16. Goding, J. W. (1996). Monoclonal antibodies: Principles and practice. London: Academic Press Inc. Limited.

17. Elvander $M$, Edwards $S$, Näslund I $S$, et al. Evaluation and application of an indirect ELISA for the detection of antibodies to bovine respiratory syncytial virus in milk, bulk milk, and serum[J]. Journal of Veterinary Diagnostic Investigation, 1995, 7(2): 177-182.

18. Pei S C, Zhang Y Y, Eremin S A, et al. Detection of aflatoxin M1 in milk products from China by ELISA using monoclonal antibodies[J]. Food control, 2009, 20(12): 1080-1085.

19. $\mathrm{Xu} \mathrm{N}, \mathrm{Qu} \mathrm{C}, \mathrm{Ma} \mathrm{W}$, et al. Development and application of one-step ELISA for the detection of neomycin in milk[J]. Food and agricultural immunology, 2011, 22(3): 259-269.

20. Berger, R. G., Mageau, R. P., Schwab, B., \& Johnston, R. W. (1988). Detection of poultry and pork in cooked and canned meat foods by enzymelinked immunosorbent assays. Journal of the Association of Official Analytical Chemists, 71, 406-409.

21. Macedo-Silva, A., Shimokomaki, M., Vaz, A. J., Yamamoto, Y. Y., \& Tenuta-Filho, A. (2001). Textured soy protein quantification in commercial hamburger. Journal of Food Composition and Analysis, 14, 469-478.

22. Tajik H, Malekinejad H, Razavi-Rouhani S M, et al. Chloramphenicol residues in chicken liver, kidney and muscle: A comparison among the antibacterial residues monitoring methods of Four Plate Test, ELISA and HPLC[J]. Food and chemical toxicology, 2010, 48(8-9): 2464-2468.

23. Quanli Yang, Ruitian Cai, Wei Xiao, Zengfeng Wu, Xia Liu, Yan $\mathrm{Xu}$, Miaomiao Xu, Hui Zhong, Guodong Sun, Qihui Liu, Qiangqiang Fu \& Junjian Xiang. (December 05). Plasmonic ELISA for Sensitive Detection of Disease Biomarkers with a Smart Phone-Based Reader. Spring Open, 7.

24. Linan Song, D. Richard Lachno, David Hanlon, Adam Shepro, Andreas Jeromin, Dipika Gemani, Jayne A. Talbot, Margaret M. Racke, Jeffrey L. Dage \& Robert A. Dean. (2016, December 15). A digital enzyme-linked immunosorbent assay for ultrasensitive measurement of amyloid- $\beta$ 1-42 peptide in human plasma with utility for studies of Alzheimer's disease therapeutics. Spring Open, 7.

25. Linan Song, Mingwei Zhao, David C. Duffy, Joshua Hansen, Kelsey Shields, Manida Wungjiranirun, Xinhua Chen, Hua Xu, Daniel A. Leffler, Susan P. Sambol, Dale N. Gerding, Ciarán P. Kelly, Nira R. Pollock. (2015, July 15). Development and Validation of Digital Enzyme-Linked Immunosorbent Assays for Ultrasensitive Detection and Quantification of Clostridium difficile Toxins in Stool. Journal of clinical microbiology, 7.
26. Elena Pérez-Ruiz. (2018, July 26). Digital ELISA for the quantification of attomolar concentrations of Alzheimer's disease biomarker protein Tau in biological samples. ScienceDirect, 7. 